\title{
LA MORT DE DIEU \\ DANS LA PHILOSOPHIE MODERNE
}

\section{La problématique}

$\mathrm{C}^{\mathrm{N}}$ 1887, Nietzsche écrivait: "L'événement le plus actuel,

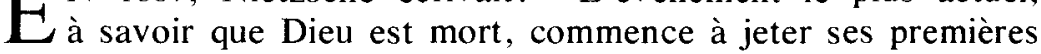
ombres sur l'Europe»'. Ceux qui regardent le monde avec attention, avec une certaine suspicion même, remarquent que le soleil s'est couché, que la confiance de jadis s'est transformée en doute, que notre monde quotidien est devenu plus sombre, plus étrange, «vieux». Cependant cet événement ne s'est pas encore annoncé publiquement, continue Nietzsche, et on ne mesure pas encore les implications de la mort de Dieu: une longue suite de démolitions, de destructions, de déclins, de renversements. Il y aura une «logique de terreur», comme on ne l'aura pas encore vu sur la surface de la terre ${ }^{2}$.

Nietzsche nous invite à considérer et la mort de Dieu et ses conséquences.

\subsection{La mort de Dieu}

La mort de Dieu est tout d'abord pour Nietzsche un événement historique auquel tout notre Occident a collaboré ${ }^{3}$. Elle signifie pour Nietzsche que Dieu et tout le divin n'est plus présent parmi nous, n'a plus d'efficacité dans notre monde ${ }^{4}$. Notre vie ne connaît plus le sacré, elle est devenue uniquement profane.

Nietzsche écrivait il y a plus d'un siècle. Aujourd'hui sa parole prend tout son sens, à l'échelle mondiale:

a) pour la première fois dans l'histoire de l'humanité l'athéisme s'organise, est devenu une organisation politique; .

b) mais un monde profane n'est pas nécessairement un monde

1 Friedrich Nietzsche, Die fröhliche Wissenschaft, par. 343, Werke in drei Bänden (Hrsg. Karl Schlechta): München, Carl Hanser, 1966, vol. II, p. 205.

Ibid.

Ibid. par. 357 (Werke, II, 227).

4 cf. Martin Heidegger, Holzwege, (Frankfurt a/M.: Vittorio Klostermann.

$1963^{4}$ ) «Nietzsche's Wort «Gott ist tot», p. 200. 
qui s'oppose à Dieu par une idéologie athée. C'est un monde où Dieu et le sacré sont absents. En ce sens les sciences modernes et la technique sont purement profanes. Nos merveilleuses sciences et techniques qui permettent de reculer les frontières de la mort, qui par leurs inventions ont élevé notre niveau de vie et nous ont permis de combattre efficacement la pauvreté, la famine et la maladie, ces sciences et techniques n'ont pas de place pour le sacré. Dans la mesure où notre société est dominée par les sciences modefnes, elle est une société purement profane. Et il s'agit là d'une négation de Dieu bien plus terrible que celle des idéologies athées. Nier Dieu implique qu'on lui accorde une grande importance. Ignorer Dieu implique qu'on ne lui en accorde aucune. En ce sens le monde européen et américain démocratique est bien plus avancé dans l'athéisme que le monde marxiste, car il vit sans Dieu tandis que le monde marxiste vit encore contre Dieu.

\subsection{Conséquences de la profanisation du monde}

Nietzsche prévoit comme conséquence de cette profanisation de la terre une longue suite de démolitions, de destructions, de déclins, de renversements, surtout une "logique de terreur».

\subsubsection{La terreur}

L'absence de Dieu et du sacré implique la temporalisation de la vie, qui n'est plus la rencontre entre le temporel et l'éternel, mais devient une succession d'événements purement temporels entre la naissance et la mort. N'ayant pas d'échappatoire au devenir, le non-croyant n'a plus de défenses contre les souffrances et la temporalité de l'histoire qui, ainsi dépourvue de sens, devient terrifiante. La terreur s'installe dans la vie quotidienne qui n'est plus qu'une marche inéluctable vers la mort. Cette terreur face à la temporalité de la vie, nous en voyons déjà aujourd'hui les premières manifestations dans la croissance de l'angoisse.

\subsubsection{La logique de terreur}

Cette terreur a sa propre logique: celle de la temporalité et du relativisme. La mort de Dieu fait sombrer notre monde dans la temporalité la plus absolue, non seulement en ce qui concerne l'histoire, mais aussi en ce qui concerne les valeurs - si Dieu n'existe pas, il n'y a plus de valeurs absolues: vérité, personne 
humaine, amour, sont autant de valeurs qui disparaissent avec Dieu. Ce qui reste, c'est l'homme dans toute sa temporalité et son organisation du monde. Et cette organisation constituera l'expression de la négation de toute vérité, de tout amour, de tout respect de la personne humaine. Ce sera un monde où avec une logique implacable et terrifiante ${ }^{5}$, toute aspiration de l'homme vers l'amour, tout respect de la personne humaine seront considérés comme une attitude maladive, et la vie humaine sera devenue une denrée périssable ${ }^{6}$.

\subsection{Cosmologies religieuses}

Pour le croyant l'histoire est la conséquence du péché, péché qui survient après la création par suite d'une faute rituelle ${ }^{7}$. La création tire l'univers du chaos et du nihil en y établissant l'ordre divin, si bien que la terre, et en particulier l'homme participent pleinement à cet ordre divin. Et puisque Dieu est éternel, le monde paradisiaque n'a pas d'histoire. L'histoire est le résultat du changement cosmique fondamental, le péché, qui a coupé la relation directe, immédiate, avec Dieu. Dès lors l'univers s'éloigne progressivement de Dieu, et à travers l'histoire, le capital divin qui y était au commencement diminue avec l'écoulement du temps. Avec l'histoire l'homme s'éloigne graduellement de Dieu et rejoint à la fin des temps le chaos originel. L'histoire est terrifiante pour le croyant car elle l'amène à son annihilation complète. Cependant il a une échappatoire. Par le rituel qui répète rituellement les actes divins, en particulier celui de la création, il peut rejoindre l'ordre divin, in illo tempore. Chaque rite représente une parti-

5 Ernst Jünger dans Ueber die Linie (Frankfurt a/M.: Vittorio Klosterman. 1950) décrit la situation nihiliste par trois caractéristiques:

a. Le nihilisme ne signifie pas le chaos, sauf comme conséquence. Au contraire, i) implique l'organisation la plus conséquente comme par exemple dans les grandes institutions de destruction où règne l'objectivité, l'hygiène et un ordre strict.

b. Le nihilisme n'a rien de maladif. En fait il implique le çulte de la santé physique et des efforts sportifs avec la négation de tout sentiment humain.

c. Le nihilisme ne signifie pas l'immoralité et peut se combiner à un philanthropisme. Seulement le nihilisme dépasse la moralité vers l'automatisme (pp. 13-20)

Les signes du nihilisme sont la réduction du monde dans toutes ses formes, et la disparition de tout ce qui fait appel au sens miraculeux (pp. 22ff.)

- Gabriel Marcel, Du refus à l'invocation (Paris: Gallimard, 1940), p. 126.

cf. Mircea Eliade. Le mythe de l'éternel retour: archétypes et répétition

(Paris: Gallimard, 1949). 
cipation de l'homme à un acte divin paradigmatique par lequel le croyant échappe à l'histoire. Par le rite il vit dans un autre temps et un autre espace. Aussi le croyant essaiera-t-il d'incorporer le plus possible les événements de sa vie à la vie sacrale, afin de les arracher à l'historicité qui à titre de conséquence du péché implique la souffrance sans sens aucun. Pour avoir de la substance, chaque acte de l'homme doit participer à l'ordre sacral. C'est le divin qui est Être, aussi le croyant cherche-t-il son être dans l'ordre divin supra-historique: "en lui nous avons la vie, le mouvement et l'être" ${ }^{8}$.

L'histoire est un devenir qui n'a de sens que s'il participe à l'ordre de l'être. Aussi une société qui exclut le sacré est une source de terreur, pour le croyant aussi bien que pour le nitzschéen. Dans cette situation, la question de Kant s'adresse à l'homme avec une force renouvelée: «que puis-je savoir, que puis-je faire, que puis-je espérer?» ${ }^{9}$. La première question, quoique purement spéculative, est fondamentale. Car l'homme espère et agit en fonction de ce qu'il sait, de ce qu'il comprend de la situation historique, et donc de ses sources.

\section{Les sources du nihilisme}

Selon Nietzsche, «nous sommes tous les assassins de Dieu» ${ }^{10}$. Comme jadis le peuple juif avait crucifié le Christ, nous avons tué Dieu en l'excluant de notre vie. Dieu est mort parce que notre civilisation ne Lui laisse pas de place. Ludwig Feuerbach, jeune hégélien, exprime la même idée: le christianisme n'a plus de place dans notre société, il est une 'idée fixe', "en contradiction flagrante avec nos assurances-vie, nos assurances incendie, nos chemins de fer et nos locomotives, avec nos pinacothèques et nos glyptothèques, avec nos écoles militaires et techniques, avec nos théâtres et nos cabinets d'histoire naturelle " ${ }^{\prime}$. Notre vie économique et technique, notre culture, notre système d'éducation et nos sciences ne s'accordent pas au christianisme. De sorte que

* Actes 17: 28. cf. aussi Eric Voegelin, Order and History, vol. I: Israel and Revelation (Louisiana State University Press, 1956), p. 3 pour l'aspect divin de l'être dans les cosmologies religieuses.

9 Immanuel Kant, Kritik der reinen Vernınft, édition B. p. 833 (ci-après: K.R.V.B. 833)

1) Die Fröhliche Wissenshaft, par. 125 (Werke II, 127)

"Sämtliche Worke (Leipzig, 1846), vol. VII ${ }^{4}$, p. 31. 
notre civilisation même est athée; il n'y a pas de place pour la foi sauf le dimanche ${ }^{12}$.

Schopenhauer, disciple de Kant et contemporain de Hegel maintient d'une manière semblable que notre monde n'a plus de place pour Dieu: «Le théisme pris au sérieux présuppose nécessairement qu'on divise le monde en ciel et terre: les hommes se meuvent sur celle-ci, Dieu, qui gouverne les hommes, se trouve en celui-là. Mais quand l'astronomie s'empare du ciel, elle s'empare aussi de Dieu: elle a agrandi le monde de telle manière qu'il n'y a plus de place pour Dieu... Il s'ensuit que le théisme doit disparaître dans la mesure où l'astronomie devient populaire». Ce que l'Église catholique a reconnu, et c'est pourquoi elle a persécuté le système copernicien ${ }^{13}$.

Nietzsche également pense que la révolution copernicienne a eu des conséquences incommensurables: "On dirait que l'homine glisse depuis Copernic sur un plan incliné - qu'il s'éloigne de plus en plus rapidement du centre - dans quelle direction? vers le néant $? . . .{ }^{14}$.

Schopenhauer et Nietzsche voient donc la source historique de notre civilisation occidentale dans le système copernicien de l'univers. Ce qui est en jeu ici ce n'est pas l'astronomie en tant que telle, mais la vision copernicienne du monde, vision qui présente deux caractéristiques: elle est basée sur la méthode hypothétique des sciences modernes, et elle implique le renversement des cosmologies traditionnelles qui laissaient les cieux tourner autour de la terre. Dans la mesure où la cosmologie traditionnelle constituait plus qu'une théorie scientifique, mais représentait une cosmogonie religieuse, son renversement par Copernic a été plus qu'un fait scientifique: il impliquait également le bouleversement des valeurs traditionnelles. Et puisque la nouvelle cosmologie s'inscrit dans le cadre des sciences modernes, la conclusion parait suivre que la vision inspirée par les sciences modernes implique un renversement des valeurs religieuses tel que notre société 'a glissé inéluctablement sur la pente du nihilisme athée. Mais dans ce cas les

12 cf. Karl Loewith, Von Hegel zı Nietzsche (Stuttgart: Kohlhammer. $\left.1958^{4}\right)$, p. 362.

13 Parerga und Paralipomena, Vol. IV, Sämtliche Werke (Hrsg. Frhr. Von Löhneysen) (Stuttgart/Frankfurt a.M.: Cotta-Insel, 1963) p. 68.

14 Genealogie der Moral, «Was bedeuten asketische Ideale?, par. 25 (Werke II, 893). 
sciences modernes ne sont pas en premier lieu responsables, car elles ont leurs bases philosophiques; la vision scientifique du monde est une vision philosophique, puisque la science en tant que telle n'a pas de vision. Mais de quelle philosophie s'agit-il? Quelle est la philosophie qui peut se prétendre copernicienne?

C'est Kant qui, explicitement, prétend avoir pensé une philosophie copernicienne. Selon Kant, la révolution copernicienne consiste en ce que Copernic n'a pas attribué les mouvements qu'on peut voir aux objets du ciel, mais aux spectateurs. Et Kant propose de construire une métaphysique sur une hypothèse semblable: "Jusqu'à maintenant on a supposé que nos connaissances devaient s'accorder aux objets. Mais toutes les tentatives, basées sur cette supposition, d'élargir ainsi a priori notre connaissance par des concepts, ont échoué. C'est pourquoi il faudrait essayer une fois si nous pourrions avancer en métaphysique en supposant que les objets doivent s'accorder à nos connaissances. ${ }^{15}$ Cette hypothèse s'applique directement à la représentation. Car si la représentation doit s'accorder aux objets de représentation, il est impossible d'avoir des connaissances a priori de ces objets. Mais si l'objet s'accorde à ma représentation, ceci devient tout à fait possible ${ }^{16}$. Kant comprend l'ordre de la réalité en termes d'organisation par le sujet. Ainsi le temps et l'espace sont des formes a priori qui nous permettent d'expérimenter le monde sous la forme d'une organisation spatio-temporelle. De même notre pensée est une activité d'organisation. Connaître la réalité signifie pour Kant «établir une unité synthétique dans la pluralité de l'expérience sensible" ${ }^{17}$. Cependant si la connaissance représente essentiellement l'application d'un ordre de concepts à une réalité qui en tant que telle n'a pas cette organisation, la connaissance se limite aussi au domaine de la réalité expérimentée et expérimentable sensiblement. L'activité de l'intelligence qui dépasse le domaine de la réalité empirique ne représente plus une connaissance proprement dite; aussi Kant distingue-t-il rigoureusement entre connaissance et pensée. «Penser un objet n'équivaut pas à le connaître. Car la connaissance exige deux éléments: d'abord le concept par lequel on pense l'objet (la catégorie), et ensuite l'intuition qui nous donne l'objet ${ }^{18}$. Il en

\footnotetext{
is K.R.V.B XVI.

16 K.R.V.B XVII.

17 K.R.V. A 150, cf. aussi B 94.

18 K.R.V. B 146.
} 
résulte que nos connaissances peuvent atteindre les objets qui transcendent notre expérience sensible, tel l'âme, le cosmos, et Dieu. Je peux penser Dieu, mais je ne peux Le connaitre ${ }^{19}$. La métaphysique ne peut prouver l'existence de Dieu. Ce qui ne signifie pas que Dieu n'existe pas, mais que la métaphysique est strictement agnostique à son sujet. Aussi l'existence de Dieu n'est-elle pas un objet de connaissance, mais de la foi ${ }^{20}$, un postulat de la raison pratique $^{21}$, et toute connaissance que je puisse avoir de Dieu est une connaissance uniquement symbolique.

Bien que selon Kant nous ne puissions connaitre Dieu au sens scientifique et philosophique du mot, la pensée de Kant n'est pas un athéisme, car l'expérience morale et religieuse nous assure que Dieu existe et qu'il est sagesse et créateur infiniment sacré et béni ${ }^{22}$. Cependant comme l'athéisme à son apothéose cesse d'être un athéisme pour devenir un nihilisme, les origines de l'athéisme ne sont pas nécessairement et apparemment athées non plus. C'est ce que Nietzsche et Schopenhauer pensaient, car l'astronomie copernicienne n'est pas athéisme en tant que telle.

En effet, la philosophie de Kant établit un dualisme de principe entre la foi et la raison, entre la théologie et la philosophie, et ce dualisme est le résultat direct de sa révolution copernicienne. Une connaissance qui organise la réalité ne peut avoir comme mesure cette réalité même. Son origine étant la spontanéité de la pensée même ${ }^{23}$, elle ne peut découvrir que ce qui est contenu dans cette spontanéité même. Aussi une telle connaissance ne peut découvrir ce qui la transcende. Afin de découvrir théoriquement Dieu, intelligence infinie, il faudrait une connaissance capable de circonscrire et de contenir la nature divine ${ }^{24}$. Cette connaissance est synthétique; elle est un modèle de la réalité qui pour s'appliquer exige une matière comme lieu de sa réalisation. Puisqu'on peut supposer que Dieu est un être supra-sensible, il n'y a pas de matière à laquelle notre concept de Dieu serait

19 Immanuel Kant, Kritik der Urteilskraft (Hrsg. Karl Vorländer, Hamburg:

Felix Meiner, 19746), p. 337.

20 Ibid. p. 343.

21 Immanuel Kant, Kritik der Urteilskraft (Hrsg. Karl Vorländer, Hamburg: Hamburg: Felix Meiner, $1974^{9}$ ) pp. 142 ff.

22 cf. Urteilskraft p. 311, Prakt. Vern. pp. 150, 181.

23 K.R.V. B 93.

24 cf. Urteilskraft, p. 311. 
applicable ${ }^{25}$. La connaissance humaine dans la philosophie de Kant est doublement agnostique: les limites de sa spontanéité intellectuelle l'empêchent de produire un concept qui puisse être adéquat à Dieu, et il lui manque toute possibilité d'expérience qui puisse servir de lieu de vérification au concept de Dieu.

\section{La philosophie poiétique}

Pénétrant plys avant dans la structure de cette connaissance copernicienne, on remarque qu'elle est connaissance hypothétique au sens où sa vérité est sa vérification, et sa source n'est pas l'expérience.

La connaissance hypothétique est une connaissance synthétique du monde. Ne représentant pas la réalité elle ne saurait être vraie que si elle se réalise dans la réalité. La réalisation du modèle est sa vérification. La vérification est le processus par lequel la réalité s'accorde au modèle. La réalité est condition, non critère de la vérité: inconnaissable en soi, elle est là comme matière pour la réalisation de la connaissance. Aussi la connaissance hypothétique est-elle un modèle de la réalité qui se réalise dans l'expérience. Un modèle qui se réalise implique la production d'un produit ${ }^{26}$. En effet, toute production impliquant une transformation de la réalité, le modèle de production ne peut représenter la réalité et ne peut avoir comme source la réalité expérimentée, si bien que toute connaissance productrice, ou poiétique, a comme point de départ une négation plus ou moins prononcée de la réalité expérimentée. Une telle connaissance n'est pas productrice parce qu'elle nie la réalité expérimentée; au contraire, elle nie la réalité parce qu'elle est productrice. Si ce n'est pas la réalité objective qui est mesure de cette connaissance, la mesure en est nécessairement la subjectivité réorganisant l'expérience pour en former un modèle de la réalité. Ce qui ne signifie pas que la connaissance poiétique est arbitraire. Au contraire, elle l'est si peu qu'elle s'accorde parfaitement à l'objectivisme le plus radical ${ }^{27}$. À titre d'organisation de la réalité elle présente le système du monde par

25 Ibid. p. 340.

26 Dans le cas de la philosophie de Kant le produit est l'expérience même: "dieses Produkt der Sinne und des Verstandes" Prolegomena zu einer jeden kïnftigen Metaphysik, par. 20 (Hrsg. Karl Vorländer, Hamburg: Felix Meiner, 1969 , p. 56).

27 cf. Martin Heidegger, Holzwege, «Die Zeit des Weltbildes», p. 81. 
la systématisation rigoureuse de la connaissance. Systématisation qui dépend, comme toute connaissance humaine, de l'expérience pour son contenu, mais qui pour son organisation, dépend de la spontanéité subjective. C'est l'inspiration qui opère la transposition idéale des données de l'expérience ${ }^{28}$.

Puisque la connaissance poiétique implique la réalisation, l'inspiration amène à une analyse des possibilités de l'inspiration en fonction de leur réalisation; cette analyse est une connaissance du possible comprenant la réalité en termes de possibilités de réalisation. Aussi affirme-t-elle le primat du possible par rapport à la réalité, comme l'affirme d'ailleurs Kant ${ }^{29}$. Ce n'est qu'en s'accordant à nos connaissances que les objets de connaissance sont réels, ce qui équivaut à affirmer que les objets sont réels dans la mesure où cette connaissance du possible se réalise.

La relation entre inspiration et analyse de sa réalisabilité est une relation dialectique, au sens hégélien du terme, entre l'idéal subjectif intérieur et sa réalisation manifeste extérieure, opposition qui exige un troisième élément comme synthèse des deux premiers.

Cette synthèse est le projet proprement dit, conclusion du raisonnement hypothétique. Le projet synthétise parfaitement l'idéal de l'hypothèse avec les possibilités de sa réalisation et ne demande rien pour sa perfection si ce n'est sa réalisation.

Cette réalisation qui est le travail du projet constitue le moment de la vérification du projet. La vérité est vérification, production de vérité, processus de réalisation.

La connaissance poiétique implique alors les moments suivants: la négation plus ou moins explicite de l'expérience comme mesure de connaissance, l'inspiration comme connaissance spontanée impliquant l'organisation idéale des données de l'expérience, la considération sur la réalisabilité de l'inspiration, le projet comme modèle concret synthétisant la réalisabilité avec l'idéalité de l'inspiration, le processus de réalisation et le produit comme réalité transformée en fonction du projet.

Cette analyse montre que la connaissance poiétique n'est ni athée ni théiste, car elle ne peut se prononcer sur Dieu puisqu'Il

28 cf. M.D. Philippe, L'activité artistique, tome I (Paris: Beauchesne, 1970) p. 252.

29 cf. Kant, K.R.V. A 287. 
transcende nécessairement tout projet humain. Ceci vaut aussi pour les différentes modalités de cette connaissance, telles les sciences modernes et la technique. De même Kant, reconnaissant les limites propres de cette connaissance, exclut Dieu de toute connaissance proprement dite.

Mais quand la connaissance poiétique devient exclusive de toute autre connaissance, elle devient implicitement athée. Au moment où la philosophie devient projet humain de la réalité, il n'y a plus de place pour Dieu. En ce sens, la philosophie de Kant est source d'athéisme. Cependant la philosophie kantienne n'est pas une pensée exceptionnelle et isolée dans la tradition moderne. En effet, toute philosophie qui identifie la connaissance philosophique de la réalité à la connaissance poiétique est implicitement ou explicitement athée. Dans la mesure où la tradition principale de la philosophie moderne conçoit la connaissance exclusivement en termes de connaissance poiétique, elle représente une vision athée de la réalité. Cette vision n'est pas dès son commencement explicitement athée. La philosophie moderne jusqu'à Hegel parle de Dieu. Ce qui ne suffit pas pour la constituer en théisme. En effet là où la philosophie poiétique parle de Dieu ex officio elle parle en réalité d'un idéal projeté par l'inspiration. C'est un idéal humain qui demande comme achèvement de soi la réalisation concrète, et cette fabrication de Dieu constitue la vérification de l'idée de Dieu. L'histoire de l'athéisme moderne comprend alors deux mouvements. Elle élimine d'abord le Dieu de la foi de ses considérations comme d'un domaine qui n'appartient pas en propre à l'inspiration poiétique de la réalité. Ensuite elle découvre graduellement son propre athéisme, elle découvre graduellement que le Dieu de la philosophie poiétique n'est que la projection d'un idéal purement humain. En ce qui suit nous toucherons les phases les plus saillantes de la philosophie poiétique athée dans l'histoire de la philosophie moderne.

\section{L'athéisme moderne}

Hegel représente une synthèse au double sens hégélien de conclusion et de point de départ de nouvelles antithèses. Son génie fut de construire une philosophie poiétique qui n'exclut aucun aspect de la réalité. Puisque la mesure de la connaissance poiétique n'est pas la réalité mais cette connaissance même ${ }^{30}$, la con-

30 Enzyklopädie der philosophischen Wissenschaften, par. 213: «denn die Wahrheit ist dies, dass die Objektivität dem Begriffe entspricht ». 
naissance ultime de la réalité (métaphysique) devient nécessairement chez Hegel connaissance de cette connaissance (logique). La philosophie de Hegel devient alors le système de cette connaissance, c'est-à-dire de l'Esprit. La réalité comprise en termes de projet qui se réalise implique la dialectique comme processus de réalisation ${ }^{31}$ et l'Esprit comme sujet de la dialectique. Aussi Hegel reproche-t-il à Spinoza et à Schelling d'interpréter l'Esprit uniquement en termes d'identité avec la réalité (substance) sans tenir compte du dynamisme exigé par la réalisation de l'Esprit même ${ }^{32}$ : "Selon ma façon de voir, qui doit seulement justifier la présentation du système, tout dépend de ce point essentiel: saisir et exprimer le vrai (i.e. la réalité dans sa totalité), non seulement comme substance, mais encore comme sujet ${ }^{33}$. La substance qui est Esprit est le devenir et ce qu'il est en soi ${ }^{34}$, c'est-à-dire sujet $^{35}$. La vie de l'esprit est action (Tat), et l'action suppose une matière qui est travaillée et transformée ${ }^{36}$. Ce travail de l'esprit, ce processus d'auto-réalisation, c'est l'histoire, par laquelle l'Esprit devient son propre produit ${ }^{37}$.

Une telle philosophie qui regarde la réalité comme projet poiétique qui se réalise à travers le travail de l'histoire ne peut plus se taire au sujet de Dieu comme l'avait fait Kant. Aussi Hegel identifie-t-il Dieu à l'Esprit et il développe une théologie où la Trinité correspond exactement aux phases de l'histoire de

${ }^{31}$ Wissenschaft der Logik (Hrsg. Georg Lasson, Hamburg: Felix Meiner, $\left.1967^{2}\right)$ Pars. I, p. 36.

32 Enzyklopädie, par. 573: «Am genauesten würden sie/die Pantheismen/ als die Systeme bestimmt, welche das Absolute nur als die Substanz fassen. ... Der Mangel dieser sämtlichen Vorstellungsweisen und Systeme ist, nicht zur Bestimmung der Substanz als Subjekt und als Geist fortzugehen."

33 Phänomenologie des Geistes (Hrsg. Johannes Hoffmeister. Hamburg: Felix Meiner, $1952^{x}$ ) p. 19.

34 Ibid. p. 558.

35 Ibid. p. 528.

36 Einleitung in die Geschichte der Philosophie (Hrsg. Johannes Hoffmeister, Hamburg: Felix Meiner, 1966 ${ }^{4}$, p. 13: «Sein/des Geistes/Leben ist Tat. Die Tat hat einen vorhandenen Stoff zu ihrer Voraussetzung, auf welchen sie gerichtet ist und den sie nicht etwa bloss vermehrt, durch hinzugefïgtes Material verbreitert, sondern wesentlich bearbeitet und umbildet."

37 Vorlesungen über die Philosophie der Weltgeschichte, vol. 1: Die Vernunft in der Geschichte (Hrsg. Johannes Hoffmeister, Hamburg: Felix Meiner. 1970") p. 72: «Der Geist ist wesentlich Resultat seiner Tätigheit : » 
l'Esprit ${ }^{38}$. Cependant le Dieu de la philosophie de Hegel n'est point le Dieu d'Abraham, ni celui, d'ailleurs, du christianisme. Hegel comprend la vie divine en termes d'histoire qui constitue un développement de Dieu ${ }^{39}$. Cette histoire comprend la négation antithétique de Dieu dans l'homme par la création. Car l'esprit étant connaissance de soi dans son autre ${ }^{40}$, Dieu doit devenir son autre. Dieu se connaissant en l'homme implique la connaissance par l'homme de Dieu, connaissance qui se développe en la connaissance de śoi de l'homme en Dieu ${ }^{41}$. Aussi l'histoire divine devient-elle l'histoire de la divinisation de l'homme par lui-même. En réalisant Dieu l'homme se réalise, si bien que le culte de Dieu est dépassé par le culte de l'Esprit et la théologie par la philosophie hégélienne ${ }^{42}$.

Pour Hegel, l'antithèse est une négation qui cache une identité profonde. Les jeunes hégéliens sont antithétiques par rapport à Hegel dans ce sens: ils mettent en lumière le dynamisme producteur de la philosophie poiétique hégélienne, ce qui a comme corollaire l'affirmation explicite de l'athéisme. Ainsi Feuerbach maintient-il que Dieu n'est que la projection de l'Idéal de l'humanité $^{43}$. Dieu est la tâche que l'humanité se donne à réaliser, et le sens de l'histoire c'est la réalisation d'une humanité idéale. Aussi l'athée est celui qui nie l'existence de Dieu, sans pour autant nier les attributs divins comme autant d'idéaux humains ${ }^{44}$. Si le christianisme maintient que Dieu est Personne et Amour, ceci signifie que la personne et l'amour est un idéal humain que l'homme projette afin de le réaliser dans l'histoire.

38 Ibid. p. 58: «Im Christentum aber ist Gott als Geist offenbart, und zwar ist er zuerst Vater, Macht, abstrakt Allgemeines, das noch eingehüllt ist, zweitens ist er sich als Gegenstand, ein Anderes seiner selbst, ein sich Entzweiendes, der Sohn. Dieses Andere seiner selbst ist aber ebenso unmittelbar er selbst; er weiss sich darin und schaut sich darin an, - und eben dieses Sichwissen und Sichanschauen ist drittens der Geist selber. "

${ }^{39}$ Ibid. p. 48: "Die Geschichte ist die Entfaltung der Natur Gottes...".

40 Phänomenologie, p. 528.

41 Enzyklopädie, par. 564.

42 En-yklopädie par. 572, 577; Geschichte der Philosophie, p. 150: «Die Philosophie ist also an sich schon eine weitere Bestimmtheit oder Charakter des Geistes, sie ist die innere Geburtstätte des Geistes, der später als Wirklichkeit auftritt. »

43 cf. Wesen des Clivistentums, vol. IV Sämtliche Werke, p. 16.: "Der Mensch verlegt sein Wesen zuerst ausser sich, ehe er es in sich findet. »

44 Ibid. p. 17. 
Marx reprend l'athéisme de Feuerbach en accentuant le lien secret entre athéisme et réalisation d'une humanité idéale. Si la philosophie devient anthropologie chez Feuerbach, elle devient idéologie pratique chez $\operatorname{Marx}^{45}$. Car seule une humanité athée pourrait réaliser l'idéal et, partant, l'épanouissement absolu de l'humanité: une humanité qui croit en Dieu, projette son idéal en Dieu, et reste passive devant Dieu qu'elle croit parfait. Aussi l'athéisme comme processus de réduction de Dieu à des dimensions anthropomorphiques est une condition sine qua non de la réalisation de l'humanité ${ }^{46}$.

L'athéisme de Marx est un athéisme basé sur la foi dans l'avenir glorieux de l'humanité ${ }^{47}$. Il revient à Schopenhauer et à Nietzsche de formuler un athéisme sans foi.

Le christianisme ne met pas en doute l'existence de Dieu, ce qui est juste, dit Schopenhauer, car Kant a montré qu'il est impussible de prouver l'existence de Dieu par démonstration. Ayant ainsi donné le coup de grâce à la théologie spéculative, Kant a essayé d'adoucir son agnosticisme en avançant des arguments moraux en faveur de la foi en Dieu. Cependant il ne s'agit pas d'une preuve de l'existence de Dieu mais d'un schéma régulateur de la morale, car Dieu n'y est considéré que comme hypothèse utile à la morale ${ }^{48}$. En effet il est impossible que le Dieu personnel du théisme existe réellement. Si Dieu a créé l'homme, il l'a créé avec toutes ses caractéristiques qui déterminent ses actions, si bien

45 «Thesen über Feuerbach», XI dans Karl Marx, Friedrich Engels, Die deutsche ldeologie (Hrsg. V. Adoratskij), Wien/Berlin: Verlag für Litteratur und Politik, 1932), 535: “Die Philosophen haben die Welt nur verschieden interpretiert, es kömmt darauf an sie zu verändern." (ci-après: MEGA, p. 535.)

46 Marx, MEGA, p. 534 (These 4): «Feuerbach geht von dem Faktum der religiösen Selbstentfremdung, der Verdopplung der Welt in eine religiöse und eine weltlich aus. ...Aber dass die weltlich Grundlage sich von sich selst abhebt und sich ein selbständiges Reich in den Wolken fixiert, ist nur aus der Selbstzerrissenheit und Sichselbstwidersprechen dieser weltlichen Grundlage zu erklären. Diese selbst muss also in sich selbst sowohl in ihrem Widerspruch verstanden, als praktisch revolutioniert werden. Also nachdem z.B. die irdische Familie als das Geheimnis der heiligen Familie entdeckt ist, muss nun erstereselbst theoretisch und praktisch vernichtet werden.»

${ }^{47}$ Pour Karl Loewith le marxisme est une hérésie du messianisme judaïque. cf. Meaning in History (Chicago: University of Chicago Press, 1958), pp. 44f.

48 Arthur Schopenhauer, Parerga wnd Paralipomena I, «Fragmente zur Geschichte der Philosophie», par. 13 «Noch einige erläuterungen zur Kantischen Philosophie» (Werke, IV, p. 132 ff.). 
que la doctrine de la création par Dieu ne peut s'accorder avec la doctrine de la liberté humaine sans laquelle on ne peut considérer l'homme comme moralement responsable ${ }^{49}$. D'autre part la doctrine de l'immortalité de l'âme ne s'accorde pas davantage à l'idée d'une création ex nihilo: comment peut-on croire qu'une existence qui a commencé il y a si peu d'années ne disparaît pas avec la mort, mais continuera miraculeusement à exister éternellement? ${ }^{50}$. D'ailleurs la doctrine de la création même ne s'accorde pas aux faits de la vie. Comment peut-on accepter que le monde soit l'œuvre d'une bonté, d'une sagesse et d'une puissance infinies si on considère que la vie n'est que nécessité et angoisse, souffrance et fardeau ${ }^{51}$ ? Loin de promouvoir le bonheur, la vie n'est que souffrance. Aussi est-ce une erreur fondamentale rendant la réalité absurde que de penser que le bonheur est la fin de la vie ${ }^{52}$. $\mathrm{Si}$ la vie est tragique, c'est que la souffrance est un processus de purification. Mourir est la seule fin de la vie ${ }^{53}$, c'est le fruit de la vie. "La mort est l'événement, le résumé ou le total de la vie qui exprime d'un coup la leçon que la vie nous a donnée successivement et partiellement, à savoir que tout l'effort dans la vie est la manifestation d'une force inutile, vaine, contradictoire, si bien que le débarras de la vie est son salut ${ }^{54}$. Objectivement parlant, la seule conclusion qui s'impose c'est que la vie est un jeu qui "n'en vaut pas la chandelle» 55 . Et pourtant chaque homme garde et défend sa vie comme si elle avait une valeur infinie; chacun aime la vie par-dessus tout et craint la mort plus que tout autre danger. Cette opposition entre la misère de la vie et l'amour de la vie ne s'explique que si l'on suppose que la vie est la manifestation d'une volonté aveugle qui agit comme instinct de $v i e^{56}$. Le principe de la réalité est la volonté, qui cherche à se donner une forme objective à travers les diverses manifestations de la nature ${ }^{57}$. Ce principe n'est pas le résultat d'une connais-

49 Ibid. pp. $152 \mathrm{f}$.

so Ibid. p. 156.

51 lbid. p. 151.

52 Arthur Schopenhauer, Die Welt als Wille und Vorstellung, vol. II, Ch. 49: "Die Heilsordnung" (Werke II, p. 813)

53 lbid. p. 817.

54 Ibid.

s5 Arthur Schopenhauer, Welt als Wille und Vorstellung vol. 11, Ch. 28: "Charakteristik des Willens zum Leben», (Werke II, p. 463).

56 Ibid. p. 464.

57 Ibid. p. $411-423$. 
sance de la vie, une conclusio ex praemissis, mais à titre de principe de la réalité, la volonté est aussi principe de toute connaissance $^{58}$. Ainsi la croyance en Dieu n'est pas le résultat d'une certaine connaissance, mais de la volonté ${ }^{59}$, qui se crée un Dieu afin de donner à la vie l'espoir de l'immortalité et d'une aide surnaturelle dans les vicissitudes de l'existence ${ }^{60}$. Préparant le nihilisme athée de Nietzsche, Schopenhauer revêt dans l'histoire de l'athéisme moderne une place particulièrement importante. La volonté est l'Être de tout ce qui est, et à titre de principe ultime de la réalité, elle détermine la connaissance. Dans le cadre de la tradition philosophique moderne l'affirmation de la volonté comme principe de réalité est la conséquence ultime d'une philosophie poiétique. La fin du projet n'est pas la contemplation mais la réalisation ${ }^{61}$. Aussi le principe du projet se sert-il de l'intelligence pour la formulation du projet. Et dans la mesure où la connaissance poiétique implique l'adaptation de la réalité au projet même, le principe du projet est ipso facto principe de la réalité. Aussi l'interprétation poiétique de la réalité voit-elle comme principe de la réalité un principe de réalisation que Schopenhauer appelle la volonté. Cette volonté est alors principe du projet et de la réalisation. C'est ainsi que la croyance en Dieu est une hypothèse avancée en fonction de la réalisation de la réalité. Cependant il s'agit d'un projet irréalisable, car si Dieu existait la vie conduirait au bonheur. Mais la fin de la vie n'est pas le bonheur ${ }^{62}$, la fin de la vie à l'époque moderne est la réalisation du projet. Puisque le sens de la vie est la réalisation sans Dieu du projet de la subjectivité humaine, il ne reste à l'homme que le pessimisme.

Chez Nietzsche la volonté devient volonté de puissance et le pessimisme devient nihilisme sans Dieu. Nietzsche constitue la fin de la philosophie moderne, au double sens de terme de la philosophie moderne et de celui qui en montre l'essence.

\footnotetext{
58 Ibid. p. 466.

59 Parerga, Werke IV, pp. 156f.

60 lbid. 147.
}

61 Ibid. 147. Russell et Scheler parlent dans ce cadre d'une connaissance pour la maîtrise du monde; cf. Bernard Russell, The Scientific Outlook (London: George Allen \& Unwin, 1931) p. 270, et Max Scheler Vom Ewigen im Menschen (Bern: Francke, $1968^{5}$ ) p. 92.

62 cf. Aristote, Éthique à Nicomaque, 1, 5 (1097 b 1): «Le bonheur semble ètre au suprême degré une fin de ce genre car nous le choisissons toujours pour lui-même et jamais en vue d'une autre chose.» 


\section{Nietzsche}

Le point de départ de la philosophie de Nietzsche est la négation de l'existence de Dieu. Le christianisme et la foi en Dieu sont en train de disparaitre car Dieu n'existe pas ${ }^{63}$. Dieu est mort et nous l'avons tué: "N'avez-vous pas entendu parler de ce fou qui, ayant allumé une lanterne en plein midi, courait sur la place du marché et criait sans cesse : «Je cherche Dieu! Je cherche Dieu !»... "Où est Dieư? cria-t-il, je vais vous le dire! Nous l'avons tué vous et moi » $^{64}$ Nous, dans la parabole de Nietzsche, ce sont les gens du marché, lieu d'expression des valeurs et des mœurs qui sont à la mode et qui constituent la civilisation. Notre civilisation est profondément athée et c'est pourquoi Dieu est mort. Heidegger se demande si en annonçant la mort de Dieu, Nietzsche ne fait pas que formuler ce que l'histoire de l'Occident a toujours sousentendu ${ }^{65}$.

Mais comment avons-nous tué Dieu? Si Dieu existe, Il est immortel et ne peut mourir. Nietzsche affirme bien que Dieu a existé, mais qu'il est mort maintenant et que notre société, avec ses compagnies d'assurance etc. a tué Dieu. Dieu est mort parce que notre civilisation l'a exclu de sa vie quotidienne. Dieu est mort parce que la foi en Dieu n'est plus une force efficace dans notre vie occidentale. La mort de Dieu dans la philosophie de Nietzsche signifie la perte, ou plus précisément, l'absence de foi en Dieu. Mais l'absence de foi en Dieu signifie plus que la négation de Dieu. Elle signifie aussi que la terre est désenchaînée de son soleil, et que l'horizon tout entier est effacé. Cet horizon, ce sont les valeurs éternelles. La mort de Dieu entraîne la disparition des valeurs éternelles et absolues ${ }^{66}$. La conséquence de la mort de Dieu est le relativisme le plus absolu, le relativisme qui est une «chute continue», où il n'y a plus ni haut ni bas. Il n'y a plus l'être, mais le devenir, en dehors duquel il n'y a rien; et le devenir n'est pas un but, il n'a aucune valeur ${ }^{67}$. Si Dieu est mort, le monde est pur devenir et le devenir n'a plus de sens. Avec la mort de Dieu, le nihilisme, le plus terrible des hôtes, frappe à la

\footnotetext{
6.3 Nietzsche, Der Antichrist, par. 38 (Werke II, 1199).

64 Nietzsche, Die Fröhliche Wissenschaft par. 125 (Werke II, 127).

65 Martin Heidegger, Holzwege, Nietzsches Wort «Gott ist tot»; p. 196.

66 Ibid. p. 200.

67 Wille zur Macht, aph. 617 (Werke III, 884); ci-après WzM 617.
} 
porte $^{68}$. Le nihilisme, c'est le sentiment pénétrant du néant ${ }^{69}$, c'est le refus radical de toute valeur et tout sens dans la vie ${ }^{70}$ à la suite de la négation de tout être, de tout ce qui est absolu, de tout ce qui est divin ${ }^{71}$. Le nihilisme, c'est vivre la vie comme une absurdité, et la forme la plus extrême du nihilisme c'est d'affirmer l'éternité du néant, de l'absurde ${ }^{72}$. Nietzsche a compris qu'un monde sans Dieu est un monde nihiliste sans vérité, sans valeur, sans espoir, qui a sa logique de terreur. Après la mort de Dieu nous errons à travers un néant infini, le vide nous souffle dans la figure; il n'y a plus que la nuit, et la nuit devient toujours plus épaisse.

Pour Nietzsche la mort de Dieu signifie l'éclipse de la race humaine telle que nous la connaissons. Afin de survivre à la mort de Dieu, il faut nécessairement que l'humanité change radicalement. La mort de Dieu inaugure l'histoire d'une humanité impie, sans Dieu ${ }^{73}$.

«Dieu est mort: maintenant nous voulons que le surhomme vive», dit Zarathustra ${ }^{74}$. Le principe de cette transformation de l'homme en surhomme se trouve dans la vie même. La mort de Dieu impliquant la négation de tout principe transcendant, le principe de la vie et de la survie doit être strictement immanent. Il faut que la vie se donne la vie; la vie doit se dépasser continuellement ${ }^{75}$. C'est l'immanence qui devient principe de la transcendance. Ce principe, Nietzsche l'appelle la volonté de puissance. La volonté de puissance est «la volonté génératrice inépuisable de la vie ${ }^{76}$. Or la volonté est principe d'action, elle est cause efficiente. Aussi le principe de vie est-il un principe dans l'ordre de l'efficacité. Et un principe n'est principe que s'il exerce sa causalité. L'exercice de l'efficacité implique la transformation et la domination de la matière sur laquelle cette cause exerce son activité. Comme le dit Nietzsche lui-même: "La vie n'est pas

\footnotetext{
68 WzM, Zum Plan (Werke III, 881).

69 Ibid. 1020 (Werke 11I, 661).

70 Ibid. Zum Plan (Werke III, 881).

71 Ibid. 15 (Werke 1II, 555).

72 Ibid. 55 (Werke III, 853).

73 Nietzsche, Also sprach Zarathustra, III: "Von der verkleinendern
} Tugend 3 (Werke II, 420).

74 Zarathustra, IV, "Vom höherem Menschen" 2 (Werke II, 523).

75 Zarathustra II, "Von der Selbstüberwindung" (Werke II, 370).

76 Ibid. cf. aussi Werke II, pp. 578, 729, 819; III, pp. 480, 854, 896. 
adaptation de facteurs internes à des conditions extérieures; elle est volonté de puissance qui depuis l'intérieur domine et incorpore toujours plus son 'milieu' ${ }^{77}$. Aussi la volonté-efficacité estelle une volonté de puissance, non au sens où elle cherche la puissance à titre d'un but à réaliser. Car la mort de Dieu implique la négation de toute finalité. La volonté-efficacité est volonté de puissance en ce sens qu'elle existe comme puissance qui s'exerce, qui à titre de domination du milieu implique l'augmentation de puissance ${ }^{78}$. C'est cette augmentation de puissance qui permettra à la vie de survivre à la mort de Dieu. À condition qu'elle soit le principe ultime de la vie et de tous les aspects de la réalité. Aussi Nietzsche comprend-il en particulier la connaissance et la technique comme manifestations de la volonté de puissance.

Nietzsche appelle la technique «art», et il en dit qu'elle a comme condition le sentiment de transport (Rausch), d'intensification de la vie. Ce sentiment peut prendre maintes formes depuis l'excitation sexuelle jusqu'aux transes sous l'influence des drogues, et les sentiments de transport dus à une volonté particulièrement intensive. Ce qui est essentiel à tous ces états de transport, c'est le sentiment d'augmentation de puissance et de plénitude. Ces états sont une source de richesse. On enrichit tout à partir de et par sa propre plénitude. "Dans cet état l'homme transforme les choses jusqu'à ce qu'elles représentent sa perfection. Ce devoir transformer en sa perfection - c'est l'art " ${ }^{79}$. Nietzsche comprend l'art en termes de transformation de la réalité par la volonté de puissance. Or la transformation est production. Et la production ainsi comprise représente la perfection de la vie car elle constitue l'augmentation de puissance. La transformation de la réalité représentant l'exercice même de la volonté de puissance, la production constitue pour Nietzsche le mode d'être de la volonté de puissance le plus authentique. C'est grâce à la production que l'homme survivra à la mort de Dieu. Et le surhomme est l'homme qui produit, l'homo faber. Aussi Nietzsche dit-il de l'art qu'il est le grand stimulant de la vie, la rédemption des savants, des gens qui agissent et qui souffrent ${ }^{80}$. Pour Nietzsche la survie de l'homme

77 WzM 681 (Werke III, 898).

78 Martin Heidegger, Nietzsche (Pfullingen: Neskel 1961) vol. I, pp. 70-79.

79 Nietzsche, Gotzen-Dämmerung, «Streifzüge eines Unzeitgemässen », par. 8 (Werke II, 995).

80 WzM 853 (Werke III, 693). 
moderne est donc intimement liée à la production. Or la généralisation de la production c'est la technique, si bien que pour Nietzsche la survie de l'humanité ne s'accomplit que par l'hégémonie de la technique. L'âge athée est aussi l'âge de la technique.

Et si la technique est le mode d'être authentique de la volonté de puissance, Nietzsche comprendra la connaissance, le savoir, la science, non seulement comme modes de la volonté de puissance mais encore comme fonction de la technique. Si la volonté de puissance est le principe de la vie, la puissance est plus importante que la vérité ${ }^{81}$, de sorte que ce qui importe au niveau de la connaissance ce n'est pas en premier lieu la vérité, mais la puissance. La vérité devient fonction de la puissance: une connaissance est vraie dans la mesure où elle affirme et reflète la puissance, si bien que le critère de vérité est l'augmentation du sentiment de puissance ${ }^{82}$. Évidemment la connaissance ne transforme pas la réalité, elle ne réalise rien. Elle n'augmente pas la puissance, mais peut donner le sentiment d'augmentation de puissance. La vérité n'est pas quelque chose que l'on prouve, que l'on découvre; la vérité est à créer. Et sa création a la fonction de rendre la réalité plus sûre; elle est une rationalisation, une systématisation du monde. La vérité organise le monde ${ }^{83}$. Ce qui implique que la vérité n'est pas nécessairement vraie. La connaissance n'est qu'une perspective que jette la volonté de puissance sur la réalité afin de s'assurer de l'augmentation de puissance. Aussi Nietzsche maintient-il que la vérité est «une sorte d'erreur sans laquelle un type déterminé d'être vivant ne pourrait vivre " ${ }^{84}$.

De même la croyance en Dieu est une fabrication, une invention de la volonté de puissance. C'est ce que St Paul a compris. Le Dieu de St Paul ne représente en réalité que la décision de St Paul $^{85}$. Dieu est une création et une fiction ${ }^{86}$. Cependant Dieu à titre de principe transcendant s'oppose radicalement à la volonté de puissance. Dieu est l'ennemi et la contradiction de la vie ${ }^{87}$.

81 Nietzsche, Menschliches, Allzumenschliches, vol. II, "Vermischte Meinungen und Sprüche», par. 226 (Werke I, 827).

82 WzM 534 (Werke III, 919).

83 WzM 552 (Werke III, 541).

84 WzM 493 (Werke III, 844).

8s Antichrist par. 47 (Werke II, 1212).

86 Werke I, 1073, 1101; II, 208, 297, 891, 943; III 588, 600.

87 Antichrist par. 18 (Werke II, 1178). 
Comment la volonté de puissance peut-elle alors produire le principe de sa propre annihilation? Nietzsche distingue à ce sujet entre deux modes de la volonté de puissance, le mode authentique, personnifié par l'aristocrate et le surhomme, et le mode de décadence, qui anime les faibles, les esclaves, le dernier des hommes. Ce dernier mode de la volonté veut le néant plutôt que de ne pas vouloir ${ }^{88}$. Le néant, dans ce cas précis, c'est Dieu, et Nietzsche voit en particulier deux situations qui donnent naissance à la croyance en Dieu: la souffrance et le ressentiment.

Puisque la volonté de puissance signifie la transformation de la réalité, et que toute transformation porte avec elle la souffrance, la souffrance est essentielle à la vie ${ }^{89}$, comme l'avait déjà affirmé Schopenhauer. Aussi Nietzsche maintient-il que plus on comprend profondément la vie, plus on comprend aussi la souffrance ${ }^{90}$. Et la capacité de souffrance détermine aussi le rang d'une personne ${ }^{91}$. Cependant si la souffrance dépasse les capacités de résistance, la vie invente un mensonge ${ }^{92}$ qui donne confiance en la vie, qui séduit.

D'autre part la croyance en Dieu réalise aussi la puissance de ressentiment. En proclamant la toute-puissance transcendante de Dieu on exige l'obéissance absolue à cette puissance. Et on s'impose soi-même comme puissance ${ }^{93}$. Aussi l'Église et les prêtres sont-ils animés selon Nietzsche par une volonté de puissance ${ }^{94}$, mais une volonté animée par le ressentiment. Ne pouvant s'imposer directement l'homme mû par le ressentiment soumet son monde entier à l'état d'esclavage ${ }^{95}$. Le Dieu des chrétiens est un

88 Genealogie der Moral, «Was bedeuten asketische Ideale?", par 28 (Werke II, 900): “-- das alles bedeutet, wagen wir es, dies zu begreifen, einen Willen zum Nichts, einen Widerwillen gegen das Leben, eine Auflehnung gegen die grundsätzlichsten Voraussetzungen des Lebens, aber es ist und bleibt ein Wille!... Und, um es noch zum Schluss zu sagen...: lieber will noch der Mensch das Nichts wollen, als nicht wollen..."

89 Jenseits von Gut und Böse, par. 225 (Werke II, 689).

90 Zarathustra III, «Vom Gesicht und Ratsel», I (Werke II, 408).

91 Jenseits ron Gut und Böse, par. 270 (Werke II, 744).

92 WzM 8531 (Werke 111, 693).

9. WZM 216 (Werke III, 568f).

94 Zur Genealogie der Moral, «Was bedeuten asketische Ideale», par 15 (Werke 11, 866).

95 Zar Genealogie der Moral, "Gut und Böse, Gut and Schlecht», par. 10 (Werke II, 782). 
Dieu de décadence et de ressentiment ${ }^{96}$. Le christianisme est né du ressentiment et Dieu est l'invention d'un esprit malade ${ }^{97}$.

Aussi le christianisme est-il destiné à disparaître. Dieu, ayant vécu, doit mourir, car la volonté de puissance s'affirme inéluctablement, même aux dépens de sa propre invention décadente ${ }^{98}$. Le reniement de Dieu est la rédemption du monde ${ }^{99}$.

Nietzsche représente dans l'histoire de la philosophie moderne le moment de la radicalisation de la philosophie poiétique. Sa philosophie représente le pari de l'humanité moderne occidentale, pari dont l'enjeu est la survie de l'homme même. Cependant le dépassement du dernier des hommes vers le surhomme représente un projet de l'homme moderne, aussi ce dépassement est-il non un dépassement de l'homme vers le surhomme, mais de l'homme vers le produit de son propre projet. C'est l'époque de la volonté de puissance, selon Heidegger ${ }^{100}$.

La réponse au subjectivisme nihiliste n'est pas le fidéisme, mais la découverte de la connaissance pré-copernicienne, comme l'ont développé Jaspers et Heidegger. Cette connaissance est vraie non par vérification mais dans la mesure où elle s'accorde à la réalité. À cette condition elle est pensée à l'Être, pensée qui appartient à l'Être. Cette pensée découvre et les limites de la subjectivité humaine et la transcendance comme principe téléologique de l'existence. De cette manière elle assume la connaissance poiétique comme moyen d'épanouissement humain.

Dirk Pereboom

\section{Université de Fribourg}

96 Antichrist, par 51 (Werke II, 1217): «in hoc signo siegte die décadence."

97 Nietzsche, Ecce Homo, «Genealogie der Moral » (Werke II, 1143).

98 Ecce Homo, «Warum ich ein Schicksal bin», par. 8 (Werke 1II, 1159): WZM 147 (Werke III, 566); Götzen-Dämmerung, "Moral als Widernatur» par. 4 (Werke II, 968).

99 Götzen-Dämmerung, «Die vier grossen Irrtümer», par. 8 (Werke II, 978).

100 Martin Heidegger, Vorträge und Aufsätze, "Ueberwindung der Metaphysik»(Pfullingen: Neske, 1954) pp. 72ff; Holzwege, "Wozu Dichter», p. 258. 